Indexaciones: Repositorio de Revistas UCR, DIALNET, Latindex, REDALYC Directorio y recolector de recursos digitales del Ministerio de Cultura de España, Directory of Open Access Journals. Diálogos Revista Electrónica de Historia ISSN 1409-469X. Número especial 2008. Dirección web: http://historia.fcs.ucr.ac.cr/dialogos.htm

\title{
¿Deconstruyendo el Estado? Reflexiones en torno a una renovación Teórico metodológica
}

\author{
Eduardo Madrigal Muñoz
}

Doctor en Historia por la Universidad de Costa Rica y la Universidad de Toulouse II-Le Mirail, investigador del Centro de Investigaciones Históricas de América Central, CIHAC-UCR. Correo electrónico edmadm@yahoo.es 


\section{Introducción:}

La presente ponencia tiene como objetivo plantear una serie de reflexiones teóricas sobre el origen de las nuevas tendencias historiográficas centradas en la utilización de la prosopografía y la teoría de las redes sociales para el estudio de las estructuras políticas de gobierno de la monarquía española del llamado Antiguo Régimen, sobre su desenvolvimiento reciente y sobre sus efectos a futuro en la comprensión de las sociedades premodernas y en nuestra concepción general del Estado y de las Ciencias Sociales.

No es la finalidad, por lo tanto, hacer solamente una presentación de los postulados de estas tendencias, sino más bien analizar sus elementos constitutivos y presentar a raíz de ello algunas intuiciones críticas.

Nuestro fin último será recibir la retroalimentación del público presente con la intención de que esta contribuya a ampliar la problemática teórico-metodológica que se plantea y de alcanzar algunas soluciones a los problemas que se presentan.

\section{Occidente y la crítica al paradigma estatal:}

Preocupado como estaba por llevar a cabo la construcción de un proyecto federal centroamericano, pocos años después de la Independencia, el prócer guatemalteco Manuel Montúfar y Coronado se expresaba de la siguiente forma de lo que estimaba era un obstáculo a la creación de un sentimiento nacional en la vieja Capitanía General:

“En primer lugar mi casa. Después mi parroquia o el barrio en que está situada mi casa. Síguese la ciudad o el pueblo en que nací. Luego el distrito en que está el pueblo, y en campo 
más extendido, la provincia y la nación. Por este mismo orden son los deberes con respecto a la defensa y al engrandecimiento local, y por el mismo se arreglan nuestras relaciones de paisanaje. $" 1$

Estas palabras, resumen a la perfección el funcionamiento de lo que algunos han llamado "la armadura social del Antiguo Régimen." 2 Ciertamente, nos hacen entrever la existencia de un sistema político muy sustancialmente diferente al actual en los años anteriores a la separación de España, y que pervivía obstinadamente aún después. Este sistema se nos presenta, en primer lugar, como una pirámide de pertenencias y solidaridades, en segundo lugar como una sociedad compartimentada en estamentos y corporaciones sociales y, en tercer lugar, como un sistema donde el poder no está organizado de manera racional ni centralizada como sucede bajo el Estado Moderno.

Y es que, durante siglos, la civilización occidental ha funcionado dentro de un esquema político dominado por una formación de gobierno denominada "Estado-Nación Moderno", la cual se caracteriza, en primer lugar, por una centralización del poder donde el Estado es el único organismo que concentra todos los medios de autoridad existentes, así como los medios de ejercer lo que Weber llamó la "violencia legítima”, es decir, los medios de coerción.

A su vez, el Estado Moderno se caracteriza por la existencia de una ley fundamental -llamada "constitución"- que le funda y le da sustento, y por una división de poderes consagrada en esta. $\quad \mathrm{Al}$ mismo tiempo, la constitución es la fuente de la que emanan todas las otras 1 Citado por Arturo Taracena, "Nación y república en Centroamérica (1821-1865)". En: Arturo Taracena A., y Jean Piel (comps.). Identidades nacionales y Estado moderno en Centroamérica, (San José: EUCR, 1995), 49.

2 José María Imízcoz Beunza. "Communauté, réseau social, élites. L’armature sociale de l'Ancien Régime" en Réseaux, familles et pouvoirs dans le monde ibérique à la fin de l'Ancien Régime, ed. Juan Luis Castellano y Jean-Pierre Dedieu (París: Éditions du CNRS, 1998) 31-66. 
leyes existentes dentro del contexto de este Estado, siendo la finalidad de estas dotar de ciertas prerrogativas a la población que cobija, con el fin de protegerla y garantizar su bienestar. Estas "prerrogativas" son lo que hoy denominamos "derechos".

En este esquema, si bien es cierto que el Estado como un todo concentra el poder y desprovee de él a toda otra instancia social, este poder se halla repartido en una serie de instituciones cuya finalidad es contrapesarse entre sí para que los medios de autoridad y coerción no estén concentrados en una sola instancia político-administrativa con el fin de evitar que tal instancia adquiera poderes omnímodos que le den la posibilidad de cometer abusos contra los derechos estatuidos de la población.

Tal sistema político, se caracteriza en la práctica, por la existencia de un amplísimo aparato burocrático que está constituido por un abanico de instituciones cuya finalidad es ejercer las funciones de gobierno del Estado sobre la población, es decir, de controlarla y cobijarla directamente. Además, este aparato burocrático -compuesto por ministerios y toda una bandada de instituciones autónomas- está organizado de manera jerárquica como una pirámide de mando, donde las instrucciones salen de las instancias más altas y son transmitidas por los mandos medios hasta los estratos más bajos del sistema, de donde son aplicadas directamente sobre la población. La existencia de esto implica también que debe haber un sistema de fiscalidad que garantice, mediante la recaudación tributaria, la obtención de fondos suficientes para financiar el funcionamiento de las instituciones de poder del Estado.

El Estado estaría dotado, así mismo, de unas fronteras dentro de las cuales se ejercería su soberanía y existiría una economía interna gerenciada por él mismo y destinada a lograr el bienestar de la población. Ambas cosas deberían ser protegidas por los poderes coercitivos -léase el ejército- con el fin de evitar posibles amenazas externas a este orden interno. Tal situación lo 
hace estar, por esencia, en eterno conflicto con sus Estados vecinos.

Finalmente, al proyecto político del "Estado Moderno" hubo de adicionársele un concepto de pertenencia e identidad manifestado a través, en primer lugar, de una cultura nacional impuesta a expensas de cualquier diversidad cultural existente a lo interno de sus fronteras y, en segundo lugar, de un conjunto de símbolos nacionales (bandera, escudo, himno nacional...) construidos por el Estado mismo y destinados a captar la lealtad de la población hacia las leyes e instituciones de gobierno.

Así pues, por al menos los últimos dos siglos, el occidente hegemónico y moderno ha funcionado bajo este esquema, el cual deriva en gran medida del pensamiento republicano de los pensadores ilustrados de la Revolución Francesa, al punto de que se llegó a pensar que era la máxima y mejor forma de organización política a la que cualquier sociedad humana podía aspirar. $^{3}$

Tal situación, ha provocado que esta civilización hegemónica haya intentado trasladar sus nociones mentales a otros contextos, no solo para entender a las civilizaciones que históricamente ha dominado alrededor del globo, sino también a sociedades del pasado de cuyo conocimiento se ha apropiado a través de las herramientas de la investigación y del pensamiento racional que también ha creado.

Según esta manera de entenderle, tal enfoque tendía a buscar ese Estado en otras sociedades que les eran contemporáneas o pretéritas, las que en realidad no se acomodaban bien a ese modelo. Se les trasladaba de manera anacrónica y por demás verticalista.

Todo esto es lo que, a grandes rasgos, podríamos denominar el "paradigma estatal" de occidente. En el plano de los estudios históricos, este paradigma tuvo como resultado la adopción

3 Héctor Pérez Brignolli, Breve Historia de Centroamérica, (Madrid: Alianza Editorial, 1985), 38 
de un enfoque de estudio de lo político donde lo que primaba era un estudio descriptivo de las leyes e instituciones del Estado al par de un intento avezado de entender en estos mismos términos a las sociedades del pasado o de esferas culturales extrañas a occidente, trasladándoles sus nociones hegemónicas.

En todo caso, todos los enfoques emanados de este paradigma absolutizaron al Estado -ya fuese para bien o para mal- como la máxima instancia de poder que cabe imaginar y como una entidad abstracta y externa, en todo caso, a los individuos que gobierna. Vertientes de pensamiento como el marxismo concibieron al estado como un mero instrumento político y superestructural de la clase dominante, destinado a apuntalar su poder sobre los medios de producción. Otros, como Emile Durkheim, plantearon que los seres humanos, por estar dotados de una conciencia similar, tendían a construir normas e instituciones de organización social con el fin de permanecer unidos y funcionar colectivamente pero que estas normas, después de ser construidas socialmente, se volvían autónomas del tejido social que les había dado origen y comenzaban a funcionar independientemente de la voluntad de sus creadores, pasando entonces a regir sus destinos de manera impersonal.

\section{La crisis de un paradigma hegemónico:}

Con todo, a fines del siglo XX este paradigma empezaría a hacer aguas como producto de lo que podríamos concebir como una generalizada crisis de civilización producida por la conjunción de varios fenómenos sociales de escala global. Primero que todo, durante los años 80, se produjo una crisis económica que acarreó la desaceleración del crecimiento económico de los años de posguerra y llevó con ello a un proceso de desencanto y cuestionamiento para 
con el modelo de desarrollo seguido hasta la fecha por las potencias hegemónicas. En segundo lugar, esto dio como resultado una crisis cultural, caracterizada por el desencanto hacia los viejos paradigmas ideológicos dominantes en la Modernidad, tales como la idea del progreso y la fe en la razón, el Estado y la economía industrial como claves para conseguirlo.

No debemos olvidar que estos son también los años en que se empezaron a sentir los efectos de la descolonización europea y de la degradación ambiental producto de siglos de depredación de la naturaleza por el industrialismo, lo que llevó a una aún mayor desilusión hacia los paradigmas que habían guiado todo esto. Son además los años que preludian el hundimiento del llamado "Socialismo Real", hecho que también acarreó un profundo desencanto con las certezas construidas desde tiempo atrás por el pensamiento ilustrado lógico-racional occidental. Las Ciencias Sociales habrían también de resentirse profundamente por estos cambios culturales y de visión de mundo.

De resultas de lo anterior, nuevas corrientes de pensamiento, pugnaces ya por hacerse oír al menos desde mayo de 1968, empezaron a hacer hincapié en la temática de los discursos sociales, las prácticas culturales, de lo subjetivo y de las construcciones individuales. Haciéndose eco del malestar generalizado ante la crisis de las certezas tradicionales y sus resultados muchas veces contraproducentes respecto a lo que se esperaba de ellas (carrera armamentista, degradación de los ecosistemas...), ${ }^{4}$ negaron la validez de la ciencia y la razón como herramientas para explicar el universo. Y llegaron incluso a denunciar al discurso científico como una narrativa que no solo no revela ninguna realidad sino que, antes bien, veían como una construcción cultural del Occidente imperialista destinada a enmascarar prácticas y estrategias de poder tendientes a someter a los seres humanos al dominio de sus estructuras políticas, tan despiadadas como opacas a su vista. ${ }^{5}$ $4 \quad$ Jacques Revel, "Presentación", en Giovanni Levi, Le pouvoir au village: Histoire d'un exorciste dans le Piémont du XVIIe siecle, (París: Éditions Gallimard, 1985), VIII-IX

5 Joyce Appleby, Lynn Hunt y Margaret Jacob, La verdad sobre la Historia, (México: 
Esto es lo que, a grandes rasgos, se conoce como "deconstruccionismo", "post-estructuralismo" o, más coloquialmente "post-modernismo."

En el plano específico de los estudios históricos, en los años 60 se había impuesto el enfoque Braudeliano y Labroussiano basado en las nociones de Historia Total y Larga Duración, que privilegiaba el estudio de las grandes estructuras sobredeterminadas por lo económico y con ello los enfoques macroscópicos de comprensión de lo social. Bajo este enfoque, se ha dicho, se invisibilizaba lo político para dar principal importancia al estudio de las estructuras económicas y sociales, a las que se veía como prioritarias por encima de los fenómenos "superestructurales" de los que lo político formaría parte.

A tono con los tiempos, en los años 70 se cuestionaría también a los grandes estructuralismos y se redescubriría al actor social, por lo que surgieron nuevas orientaciones en los estudios históricos. Los Post-estructuralistas (también llamados post-modernos) debilitan por su parte la posición dominante del paradigma de Braudel (también llamado enfoque Analista de la Historia).

Las críticas de estos pensadores llamados "posmodernos," con su énfasis en lo particular, en lo subjetivo y en el relativismo cultural, han ejercido un efecto transformador sobre las nociones que se tenían establecidas para abordar y entender lo social y lo político. Han traído, sin duda, como consecuencia, la deconstrucción de una serie de nociones tradicionalmente manejadas por la Historia Social como sus grandes sistemas estructurales. Por ejemplo, a la luz de las críticas de estos pensadores, los historiadores han planteado cambios sustanciales a la noción de

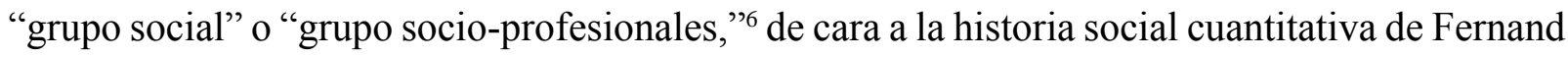

Editorial Andrés Bello, 1986), 227-230

6 Michel Bertrand (coord), Configuraciones y redes de poder: un análisis de las relaciones sociales en América Latina (Caracas: Fondo Editorial Tropikos, 2002), introducción; Simona Cerutti, "La construction des catégories sociales", en Passés Recomposés (París: Gallimard, 
Braudel y sus seguidores. Estos replanteamientos han tenido que ver con una renovada atención a los criterios jurídicos de la época para definir la pertenencia a un grupo y no solo a los socioeconómicos, extraídos de las nociones de las Ciencias Sociales actuales, que los diseñaron para entender las sociedades de clases del capitalismo industrial. Esto ayuda a no meter dentro de una misma categoría social a personajes que en la época quizá se habrían visto como distintos (o viceversa) y habrían actuado en consecuencia de esa diferencia que ellos mismos creaban y percibían por sus criterios culturales.

También la noción de mercado, para citar otro ejemplo, ha recibido cuestionamientos serios. $^{7}$ Se ha planteado que, en este tiempo, no era el espacio de transparencia y competencia perfecta que proponían los clásicos, sino que las dinámicas de intercambio de bienes y servicios -con el consecuente proceso de fijación de los precios- en realidad dependía no de fuerzas "invisibles" de oferta y demanda, sino de relaciones personales y del concepto cristiano de la caridad. Así pues, los nuevos enfoques de la Historia Económica tienden a plantearse al mercado más como un conjunto de redes de relación que, en su juego de intereses, como un conjunto de relaciones interpersonales bien tangibles que preside los intercambios, más que como el conjunto de relaciones abstractas concebidas por los clásicos.

¿Porqué no habría también el concepto de Estado de ser atacado en tal contexto? El distanciamiento con viejos enfoques de la Historia Política que consistían en una descripción extensiva de los aparatos de leyes e instituciones y también la crítica al pensamiento de Durkheim -que hemos tenido ocasión de comentar-, son solo dos de los componentes de todo un proceso de 1997), 224-233.

$7 \quad$ Giovanni Levi, Le pouvoir au village: Histoir d'un exorciste dans le Piémont du XVIIe siècle (París: Gallimard, 1985); Zacarías Moutoukias, "Peut-on faire l'économie d'une économie politique?", en Anales HSC (noviembre-diciembre 2001) nº, 1111-1128; Nicolás de Broens, Monarquia y capital mercantil: Felipe IV y las redes comerciales portuguesas (1627-1635), (Madrid: Ediciones de la Universidad Autónoma, 1998). 
replanteamiento de nuestra comprensión del funcionamiento de los aparatos de gobierno en las sociedades premodernas. Las posiciones críticas de pensadores como Michel Foucault y Pierre Bourdieu a este respecto, son bien conocidas. Los mecanismos "tecnopolíticos" de sujeción del individuo al poder y las construcciones simbólicas tendientes a apuntalar las jerarquías sociales han sido, entonces, enfatizadas por estos autores. Con esto, la visión antigua del Estado como monolito idéntico a sí mismo, autosuficiente, suprapersonal y externo a la sociedad es seriamente desmantelada y puesta por tanto en cuestión.

Todo esto es la antesala de la aparición de toda una nueva concepción de la organización política de las sociedades pre-modernas.

\section{La política en el Antiguo Régimen: abordajes novedosos}

Así pues, nuevas corrientes historiográficas han venido descubriendo que, en las sociedades anteriores al advenimiento de la así llamada "Modernidad" predominaron categorías simbólicas y construcciones jurídico-políticas y sociales radicalmente distintas a lo que el ser humano moderno está acostumbrado a tener entre sus vivencias, cuestionando así la omnipresencia del "paradigma estatal" que el pensamiento de los últimos siglos había impuesto como paradigma hegemónico.

Como dijimos, ya desde la década de 1970 el pensamiento de Michel Foucault se había encaminado a una negación de las estructuras y a una afirmación, en contrapartida, del papel actuante del actor social frente a las estas. ${ }^{8}$ Luego, en los 80 s, autores como F. Furet le devolvieron la importancia a lo político, otros como F. Dosse lanzaron duras críticas contra los

8 Bertrand, Michel (coord). Configuraciones y redes de poder: un análisis de las relaciones sociales en América Latina. Caracas: Fondo Editorial Tropikos, 2002, p.6 
Annales y su visión macroestructural de las sociedades humanas, ${ }^{9}$ al tiempo que B. Lepetit planteó un "viraje crítico" hacia una "teoría de la acción."10 Otros han señalado que el poder es un conjunto de relaciones dadas por la dinámica de los vínculos sociales de quienes en estas relaciones participan. ${ }^{11}$

Del mismo modo y en gran medida como resultado de todo este proceso, trabajos recientes han retomado el estudio histórico de lo político para darle un nuevo enfoque, a la luz de las críticas y nuevos planteamientos surgidos a raíz de estas. Por ejemplo, algunos han estudiado los discursos políticos del pasado con el fin de desmitificarlos y de descubrir las construcciones ideológicas que subyacen detrás de ellos, dando como resultado una comprensión totalmente novedosa del pensamiento y de las vivencias políticas de las organizaciones políticas anteriores al Estado Moderno. ${ }^{12}$

Pero el enfoque que más nos interesa a nosotros es el que aparece en los años 80 en Italia, caracterizado por el uso de la prosopografía y la teoría de las redes sociales. Este ha planteado toda una revisión del Antiguo Régimen como sistema de gobierno, haciendo hincapié en los lazos sociales que constituyen lazos políticos, apuntando a ofrecer una visión de la construcción de los sistemas de poder a partir de las micropartículas que los constituyen.

Este enfoque surgió de un movimiento detonado por un grupo de historiadores italianos

9 Bertrand, "Configuraciones", introducción.

10 Bernard Lepetit, "Histoire des pratiques, pratique de l'histoire" en Les formes de l'expérience (París: Albin Michel, 1995), 20

11 Zacarías Moutoukias y Jean-Pierre Dedieu, "Introducción”, en en Réseaux, familles et pouvoirs dans le monde ibérique à la fin de l'Ancien Régime, ed. Juan Luis Castellano y JeanPierre Dedieu (París: Éditions du CNRS, 1998),.8-15

12 Annick. Lempérière, Entre Dieu et le roi, la république (Paris: Les Belles Lettres, 2004); François-Xavier Guerra, Modernidad e Independencias, (Barcelona: MAPFRE, 1992); Jean-Frédéric Schaub, "El pasado republicano del espacio público" en François-Xavier Guerra y Annick Lempérière, Los espacios públicos en Iberoamérica: ambigüedades y problemas. Siglos XVIIIXI,.(México: Fondo de Cultura Económica, 1998), 27-53. 
aglutinados alrededor de la revista "Quaderni Storici", que realizaron todo un nuevo planteamiento consistente en reducir la escala del análisis social con el fin de privilegiar al actor sobre la estructura. Tal enfoque recibió desde entonces el nombre coloquial de "Microhistoria" y se basó en el uso de la metodología prosopográfica y en el enfoque teórico de redes sociales.

Con ello, estos historiadores perseguían los mismos fines de la Historia Social, es decir, llegar a una comprensión racional de las sociedades humanas mediante la aplicación de una metodología científica a su análisis, pero planteando un rechazo a los métodos cuantitativos como único medio para hacerla y un método de "jugar con las escalas" del análisis -pasando de lo micro a lo macro- para aclarar la complejidad del juego social y llegar al nivel de lo puntual.

La prosopografia fue desarrollada en el siglo XIX por historiadores de la Antigüedad -particularmente alemanes- para estudiar el Imperio Romano, como una respuesta a la acuciante estrechez de las fuentes con que se cuenta para estudiar las sociedades del mundo antiguo. ${ }^{13}$ Consiste en la creación de una biografía colectiva de un grupo social específico, generalmente delimitado en función de su pertenencia a una institución o agrupación social determinada, o sea, con base en una definicion jurídica y socioprofesional, a través de la recolección sistemática de datos sobre aspectos exteriores y medibles del grupo tales como su origen, carreras vitales, patrimonios económicos, actividades sociales, etc. El objetivo de tal práctica, en sus inicios, era aprehender el funcionamiento del aparato de gobierno romano, tratando de deducirlo de la información existente sobre aquellos que estuvieron a su servicio o sea, situándolo dentro de su medio social..$^{14}$ La adopción de esta metodología ha implicado resucitar otras que se creían ya viejas y superadas, como la biografía y la genealogía que fueron, sin embargo, revitalizadas como herramientas al servicio de la Historia Social.

13 Bertrand, Grandeur et misère..., pp.1-2

14 Bertrand, op. cit., pp. 3-5 
La conceptualización de red social apunta, por su lado, a entender la sociedad como una inmensa red de relaciones entre los actores sociales que la constituyen. Dentro de ella, circula toda una plétora de recursos que los actores deben usar para el logro de sus fines. Pero, al ser estos recursos limitados, ello determina una desigualdad de posiciones de los actores dentro del sistema y una competencia por el acceso a los recursos lo cual origina que la configuración de las relaciones existentes en la red esté en constante cambio en función de las luchas diarias de los actores por utilizarlos para mejorar su posición dentro del sistema. El sistema reticular determinaría, así, todo un conjunto de presiones y oportunidades que se abren ante los actores para que estos logren o no su deseado éxito social, dando lugar con ello al surgimiento de un conjunto de estrategias desplegadas por estos para colmar estos objetivos. Del conjunto de las estrategias elaboradas por los actores en su lucha diaria por sobrevivir y mejorar su posición dentro del sistema surgirían las estructuras de nivel macro que caracterizan el funcionamiento de la sociedad. ${ }^{15}$

Este enfoque ha sido instrumental para reconceptualizar los estudios históricos del poder pues da primacía a las estrategias de los actores para hacer y cambiar las estructuras, integra las acciones individuales con el marco estructural y no excluye lo cultural y discursivo.

El recurso a estos nuevos enfoques ha permitido desbloquear el entendimiento de los lazos políticos y sociales del Antiguo Régimen así como entender las dinámicas políticas de la época.

Su aporte esencial ha sido insistir sobre la esencial heterogeneidad de la armadura política premoderna frente a lo que entendemos modernamente como "Estado." Plantean que hay que entenderla en términos de alteridad de cara a nuestros conceptos actuales de lo político. Así, las 15 Lazega, Emmanuel. Réseaux sociaux et strutures relationnelles. París. PUF, 1998; Barabási, Albert-László. Linked: the new science of networks. Cambridge: Perseus Publishing, 2002. 
nuevas perspectivas están más dirigidas a estudiar problemáticas socio-políticas que a hacer una historia institucional tradicional, inclinada -como lo estaba la anterior- a enfatizar la descripción pura y simple de leyes e instituciones. ${ }^{16}$

La conclusión que, de manera general, han sugerido estos estudios recientes, es que la "armadura social" del Antiguo Régimen, ${ }^{17}$ estaba constituida por una plétora de corporaciones sociales como estamentos, cabildos, gremios de artesanos, cofradías, órdenes monásticas, etc. De ellas, se afirma, las monarquías dependían para poder gobernar en vista de su carencia de mecanismos directos para poder llegarle a la población. Formas de solidaridad como los grupos definidos por un estatuto personal (nobleza, clero, órdenes militares, etc.), los cuerpos y comunidades territoriales (la casa -primero y más universal de todos los cuerpos-, el pueblo, la ciudad, la provincia, el reino), las comunidades de trabajo (como gremios y consulados) y las comunidades religiosas (la parroquia, las cofradías, los conventos y las órdenes monásticas), entre otras, constituían en este tiempo coordenadas en las cuales los individuos se situaban y encontraban su lugar en el mundo.

Tal abanico de corporaciones y comunidades determinaba una multiplicidad de sentidos de pertenencia de los individuos que se asociaban a ellas, siempre de acuerdo a un principio de integración/dominación y a otro de protección/sumisión en donde para ser alguien de respeto y para ser protegido, había que estar conectado a un cuerpo colectivo y jamás existir en soledad. ${ }^{18}$ Por ello, la incorporación a este tejido socio-político pluricéntrico era un hecho vital forzoso: todo hombre debía formar parte de al menos una corporación para estar integrado a la sociedad. En el Antiguo Régimen nadie estaba solo.

16 Piénsese, por ejemplo, en los trabajos de José María Ots Capdequi, J.H. Parry y Clarence Haring, entre otros.

17 Para usar el término de José María Imízcoz.

18 Castellanos y Dedieu, cap. 1 
Por eso mismo implicaban una manera totalmente diferente -aliena- de concebir y de ejercer el poder. Implicaban la existencia de una dispersión del poder, de una "poliarquía” por seguir citando al filósofo alemán, implicaban la existencia de "poderes" más que de un único "poder."

Así pues, la monarquía española de la época aparece ante nuestros ojos como un sistema donde la corona no tenía mecanismos para llegarle directamente a la población, por lo que tenía que pasar por la mediación de un conjunto de cuerpos sociales independientes. Estos eran gobernados por el rey a través de una dinámica de intercambio de favores contra servicios en una intrincada red de compromisos políticos donde el rey necesitaba de las elites locales para gobernar y a cambio de ello les concedía favores como títulos de nobleza, venta de cargos, dispensas matrimoniales y apoyo en todo lo que fuera de su interés, desde legislar a favor de sus intereses económicos hasta favorecerles en procesos judiciales.

\section{El nuevo enfoque: aportes y críticas}

Así pues, permítasenos hacer una serie de reflexiones teóricas. Por un lado ¿qué es un estado sin su gente? ¿Acaso un grupo de edificios magestuosos donde se supone residen las instancias administrativas? ¿O más bien un conjunto de leyes e instituciones de gobierno abstractas, creadas por la sociedad pero externas a ella, que se las da a sí misma para construirla y dominarla y que pueden bien no existir más que en el papel y no tener consecuencia real? ¿Cómo tenemos que entender, entonces, cuando sus normas no se cumplen? Ya de antiguo el gran historiador francés Charles Seignobos afirmaba que el Estado y la Iglesia no existen sino por sus agentes. ${ }^{19}$ Por otro lado y en consecuencia ¿será más bien que el estado es un conjunto de 19 Gérard Noiriel, Sur la “crise” de l’Histoire. (París: Éditions Bélin, 1996), 196 
individuos y grupos sociales que, en sus relaciones y transacciones diarias construyen las normas sociales a las que van transformando y adaptando incesantemente según las direcciones que tome la corriente de sus intereses, negociaciones y relaciones de fuerza? Es aquí donde residen los aportes -pero también las críticas- que se desprenden del enfoque de redes sociales.

Un estudio puramente estructural del objeto "Estado" tiende naturalmente a enfatizar lo exterior: si consideramos al Estado como una "estructura" política, naturalmente esto nos lleva a preguntarnos cuáles son los elementos constitutivos de esta "estructura." La respuesta lógica sería, pues, que el Estado es un conjunto de leyes e instituciones, construidas de acuerdo a un tejido subyacente de ideas políticas. Esta visión tradicional del Estado asume entonces que este es un monolito y que, por su propia definición, la población bajo su control asimila automáticamente sus postulados ideológicos. En suma, como hemos ya comentado, un enfoque así tiende a no explicar verdaderamente los mecanismos mediante los cuales el aparato político-ideológico consigue efectivamente ser interiorizado por la población. Ello desemboca, naturalmente, en una historia puramente institucional. En consecuencia, una visión de las estructuras institucionales como estructuras objetivas extermina a los actores y les niega toda participación, toda voz y toda capacidad de construir su realidad e influir sobre ella.

En cambio, en los nuevos planteamientos basados en el estudio de unidades sociales restringidas, estos mecanismos son resaltados inmediatamente: eran las lealtades sociales del Antiguo Régimen las que transmitían los valores dominantes y los hacían interiorizar a la gente: la familia, la comunidad, las solidaridades de estamento, los espacios de asociación seculares y religiosos, entre otros, creaban lazos que mantenían unido al Imperio Español y que legitimaban su sistema. Un estado unitario, solo basado en sus medios de fuerza y sus constructos jurídicopolíticos no hubiera bastado de por sí para tan titánica tarea, dicho de otro modo, no podría 
haber hecho funcionar sus postulados sin contar con los mecanismos sociales para "pegar" lo local con lo global. De ahí la gran confianza de la monarquía católica en las elites locales y su apoyo irrestricto a sus costumbres de funcionamiento: tenía forzosamente que apoyarse en su gente y su gente funcionaba de acuerdo a pautas culturales precisas y conocidas, de manera que las ideologías y su articulación con el poder no se explican por la simple sujeción a un sistema formal de normas, sino por la configuración cambiante de los contactos entre individuos. Esto es también explicativo del papel desmesurado de la vida religiosa como puntal de la vida política en este tiempo.

Por otra parte, el estudio de espacios sociales reducidos -como la familia y la instituciónha posibilitado ver ámbitos sociales en donde circulaban recursos que siempre revertían para beneficio de quienes los monopolizaban, para cumplir la finalidad de reproducir su poder y posición, y con ello de guardar el orden social establecido. He aquí toda una nueva visión donde, como ha sido dicho, las instituciones políticas dejan de ser entidades puramente administrativas, impersonales y por demás neutras -cosa que el hombre de la Modernidad está acostumbrado a creer que son-, y pasan a ser vistas como entidades estratégicas. Las estrategias sociales de los actores para usar su capital social -la racionalidad instrumental con que funcionaba esta sociedad-, pasa de esta manera a ocupar el lugar de prioridad y con ello se ponen de manifiesto las variables culturales que están en la raíz la circulación de los recursos en ella.

Hay en todo esto, ciertamente, una influencia renovada de la antropología cultural, que hace saltar al primer plano conceptos como las estructuras del parentesco, el intercambio de mujeres, la reciprocidad, los sistemas de creencias (valores, culturas políticas), los "roles" e "interacciones" sociales que estructuran los procesos de construcción social de sentido, en fin, las prácticas y los rituales. Esta es precisamente la fuente del renovado interés por el estudio de 
los contextos en su multiplicidad (al reconstruir la carrera de cada individuo, hay mil visiones de las estructuras), que pone de relieve la complejidad de lo social, de la importancia de las pertenencias plurales y de su construcción, que no solo se visibiliza, sino que salta al primer plano, así como lo hace el sentido de la "alteridad," de manejar una conciencia clara de "el otro" frente al "yo." Todo revierte en la reivindicación de la capacidad de acción del actor según su posición e interacciones en el contexto social que le es dado y los recursos materiales y simbólicos con que cuente.

\section{Conclusión:}

Así, podemos concluir que, en un afán por renovar los estudios históricos purgándolos de las limitaciones del pasado y de adaptar la Historia a la nueva época, respondiendo a las críticas que se le han hecho, lo que opera en el fondo de los nuevos enfoques es una deconstrucción del Estado, pues según su punto de vista este deja de ser un monolito de leyes e instituciones y se convierte en un conjunto de individuos en configuraciones perpetuamente cambiantes cuyos movimientos y estrategias diarias producen una estructura eternamente dinámica en cuya elaboración participamos todos. 

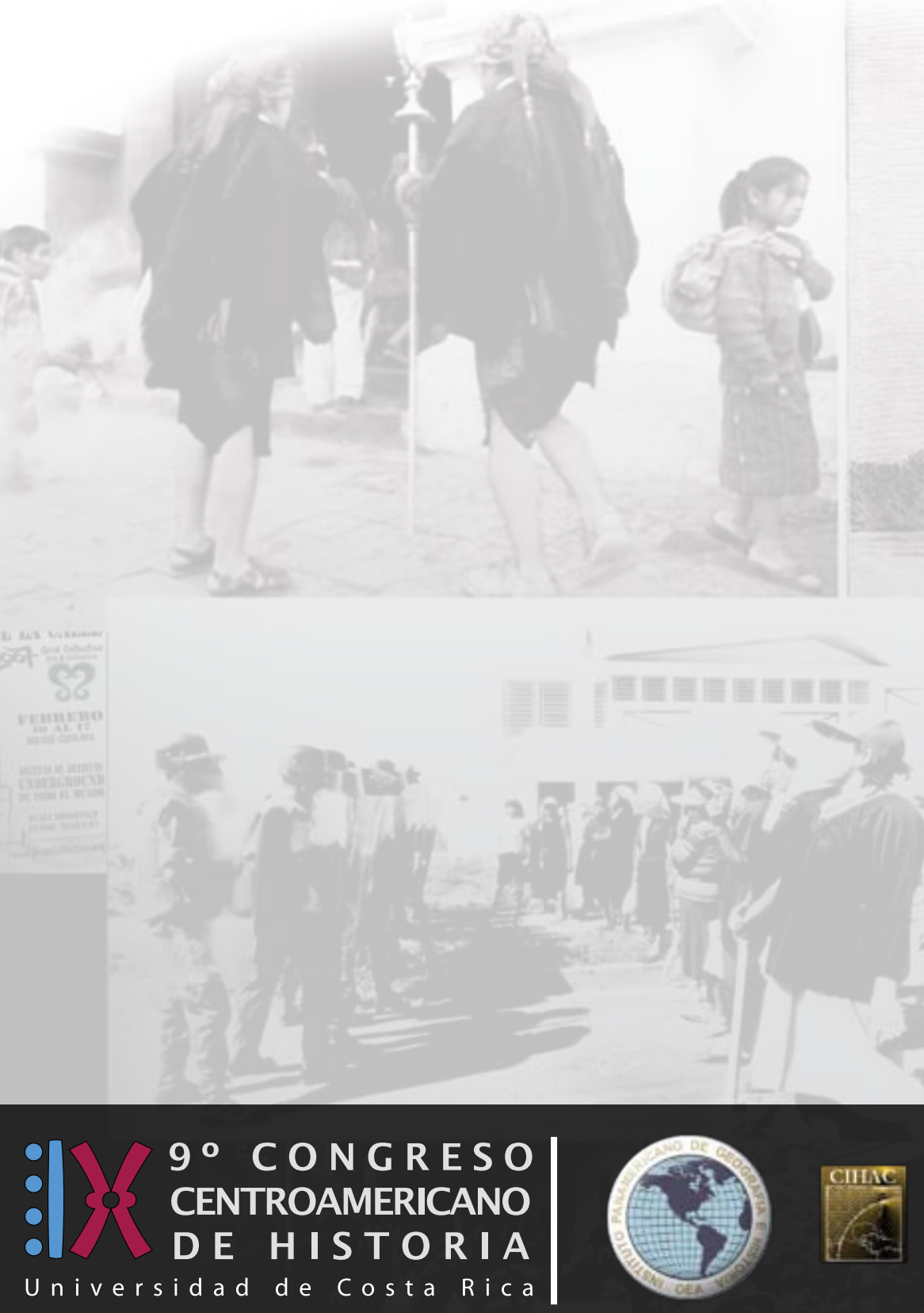